OPEN

SUBJECT AREAS:

ANIMAL PHYSIOLOGY

COEVOLUTION

ANIMAL BEHAVIOUR

BEHAVIOURAL ECOLOGY

Received

14 May 2013

Accepted

25 July 2013

Published

13 August 2013

Correspondence and requests for materials should be addressed to

K.V. (kim.valenta@ utoronto.ca.)

\section{Colour and odour drive fruit selection and seed dispersal by mouse lemurs}

\author{
Kim Valenta' ${ }^{1}$, Ryan J. Burke' ', Sarah A. Styler ${ }^{2}$, Derek A. Jackson ${ }^{2}$, Amanda D. Melin ${ }^{3}$ \& Shawn M. Lehman ${ }^{1}$ \\ 'University of Toronto, Department of Anthropology, ${ }^{2}$ University of Toronto, Department of Chemistry, ${ }^{3}$ Dartmouth College, \\ Department of Anthropology.
}

Animals and fruiting plants are involved in a complex set of interactions, with animals relying on fruiting trees as food resources, and fruiting trees relying on animals for seed dispersal. This interdependence shapes fruit signals such as colour and odour, to increase fruit detectability, and animal sensory systems, such as colour vision and olfaction to facilitate food identification and selection. Despite the ecological and evolutionary importance of plant-animal interactions for shaping animal sensory adaptations and plant characteristics, the details of the relationship are poorly understood. Here we examine the role of fruit chromaticity, luminance and odour on seed dispersal by mouse lemurs. We show that both fruit colour and odour significantly predict fruit consumption and seed dispersal by Microcebus ravelobensis and $M$. murinus. Our study is the first to quantify and examine the role of bimodal fruit signals on seed dispersal in light of the sensory abilities of the disperser.

$\mathrm{P}$ lant reproduction often requires animal vectors to provide seed dispersal or pollination services ${ }^{1}$. Numerous studies have demonstrated that plant signals and cues are critical to fruit selection by animals ${ }^{2-5}$. While ripe fruit signals refer to traits such as colour and odour that are maintained by natural selection because of their ability to reliably convey information to other organisms $s^{6}$, ripe fruit cues refer to traits that evolved in a context unrelated to animal signalling (e.g. red anthocyanine pigmentation), that may nonetheless convey reliable information to dispersers ${ }^{7}$. The question of whether plants have evolved to maximize signal detectability to potential pollinators and dispersers is contentious ${ }^{1,8}$, as is the question of how much variation in frugivore sensory phenotypes is driven by fruit signals versus cues?

Plant signals and cues available to animals depend critically on three complex factors: first, the complete signal being broadcast, second, its arbitration by the local environment ${ }^{6}$, and third, animal sensory phenotypes, which mediate the detectability of plant signals and cues to potential seed dispersers and pollinators ${ }^{10}$. Plant signals and cues are highly variable ${ }^{8}$, and comprise visual components - chromaticity (hue, saturation) ${ }^{11}$, brightness or luminance ${ }^{12}$ - and odour components, including individual volatile compounds as well as overall odour plume $\mathrm{e}^{13}$. Animal colour vision phenotypes are also highly variable, and for terrestrial vertebrates range from monochromacy to tetrachromacy ${ }^{14}$. In the case of odour-detection ability, few studies have sampled olfactory receptor (OR) gene repertoires ${ }^{15-17}$, although evidence from experimental studies and broad neuroanatomical measures indicate high variation amongst vertebrates ${ }^{18,19}$. Ideally, to determine the extent of the mutualism between animal sensory phenotype and fruit signals, models should include quantitative measures of signals as well as the ability of animals to discern those signals ${ }^{10}$. Recent studies have quantified certain fruit signals and compared them to seed dispersal ${ }^{8}$, and quantified disperser phenotypes in light of fruit colour ${ }^{11,20}$. For example, recent studies of the role of plant colour signals and cues in primate foraging decisions indicate that at least some variation in primate foraging efficiency and preferences results from variation in individual colour vision phenotypes ${ }^{11,12,21}$. Additionally, a recent study examining the relative sensory reliance in three strepsirrhines emphasized the role of vision over olfaction for diurnal lemurs ${ }^{22}$. However, to date, no study has yet quantified and qualified fruit chemistry in combination with quantitatively measuring luminance and chromaticity in light of animal-specific sensory phenotypes.

Several studies have established the importance of primates as seed dispersers ${ }^{23,24}$. Primates comprise between $25 \%$ and $40 \%$ of frugivore biomass in tropical forest $\mathrm{s}^{25}$, and defecate or spit large numbers of viable seed $\mathrm{s}^{26}$, which makes them particularly well suited to be effective dispersers. Seed dispersal by primates is critical to the maintenance of fruiting tree populations, and has been shown to contribute to the maintenance of biodiversity in tropical forests ${ }^{27}$. The case of Madagascar is particularly compelling, as primates comprise most of the seed dispersing species in those forests - indeed, only ten non-primate species in Madagascar have been identified as seed dispersers ${ }^{28}$, which is in stark contrast to the diverse disperser assemblages of other tropical biomes ${ }^{29}$. 
Understanding the relationship between endemic fruit signals and seed dispersal by Microcebus spp. is important in the heavily disturbed forests of Madagascar. The potential for Microcebus spp. as critical seed dispersers in the uniquely depauperate frugivore communities of Madagascar has recently been highlighted ${ }^{29}$. While numerous studies have demonstrated the importance of fruit morphology, including colour and size, in diurnal primate foraging decisions ${ }^{21}$, data are lacking on morphological characteristics of fruits consumed by nocturnal primates. This is the first study to compare quantitative and qualitative measures of fruit odour, as well as quantitative measures of luminance and chromaticity on fruit consumption by an animal in light of species-specific sensory phenotypes. Additionally, this is the first study to quantify fruit chromaticity and luminance for a nocturnal primate.

Here, we determine whether seeds of endemic plant species are dispersed by wild-trapped Microcebus ravelobensis and M. murinus held in short-term captivity in northwestern Madagascar, as evidenced by the presence of intact seeds in feces. We then compare chromaticity, luminance and odour of dispersed and non-dispersed species. Microcebus is an ideal taxon within which to measure the interplay between fruit cues and sensory phenotypes because their capacity for colour discrimination can be accurately modelled based on known peak cone spectral sensitivities and optical morpho$\log y^{30,31}$. Additionally, Microcebus spp. have been shown experimentally to be able to reliably distinguish olfactory cues, and retain extensive neuroanatomical structures associated with enhanced olfactory discrimination, including moist rhinaria and large olfactory bulbs $^{19,32}$. Unlike some other nocturnal primates, Microcebus spp. have retained dichromatic cone function ${ }^{30}$, which may indicate purifying natural selection acting to maintain colour vision ${ }^{33}$. Therefore, we predict that fruits that are consumed and dispersed will have a greater chromatic contrast than fruits that are not consumed. Because dichromatic animals have been shown to respond to luminance cues $^{34}$, we predict that consumed fruits will have a greater luminance contrast than unconsumed fruits. Because Microcebus $s p p$. are strepsirrhines with highly retained OR repertoires and large olfactory bulbs ${ }^{19}$ we predict that dispersed fruits will emit greater overall volatile organic compounds (VOC) than fruits that are not consumed or dispersed by Microcebus spp. and that Microcebus-dispersed fruits will be characterized by emission of similar chemical compounds. All of our analyses are one-tailed due to the directionality of our predictions.

\section{Results}

The luminance contrast between fruits and background leaves is similar for both dispersed and non-dispersed species, as indicated by their large degree of overlap (Fig. 1). Thus, contrary to our predictions, the effect of luminance on seed dispersal is not significant (Wald chi-square $=0.456, \mathrm{df}=1, \mathrm{p}=0.245$, one-tailed). Our prediction that Microcebus spp. disperse species with a higher chromatic contrast is, however, supported (Wald chi square $=3.018, \mathrm{df}=$ $1, \mathrm{p}=0.041$, one-tailed). Fruits of dispersed species have a higher chromatic (blue-yellow) contrast with background leaves than fruits of non-dispersed species (Fig. 1). Our prediction that Microcebusdispersed fruits are characterized by emission of similar volatile compounds is not supported: fruit species dispersed by lemurs do not show a significant difference in VOC compound distributions (Wald chi-square $=4.332, \mathrm{df}=5, \mathrm{p}=0.252$, one-tailed) relative to non-dispersed species (Wald chi-square $=2.682$, $\mathrm{df}=4, \mathrm{p}=0.306$, one-tailed). Our prediction that Microcebus-dispersed fruits emit greater overall VOCs than non-dispersed fruits is supported ( $\mathrm{F}=$ $8.001, \mathrm{p}=0.014$, one-tailed). Five of the six species with the lowest overall VOC emissions (integrated chromatogram area $<15,500$ ) are non-dispersed. In contrast, eight of the nine species with VOC emissions with an integrated chromatogram area $>15,500$ are dispersed (Fig. 2).

\section{Discussion}

Our prediction that fruit luminance contrast predicts fruit consumption and seed dispersal was not supported. Conversely, we did find support for the importance of chromatic contrast. Previous studies

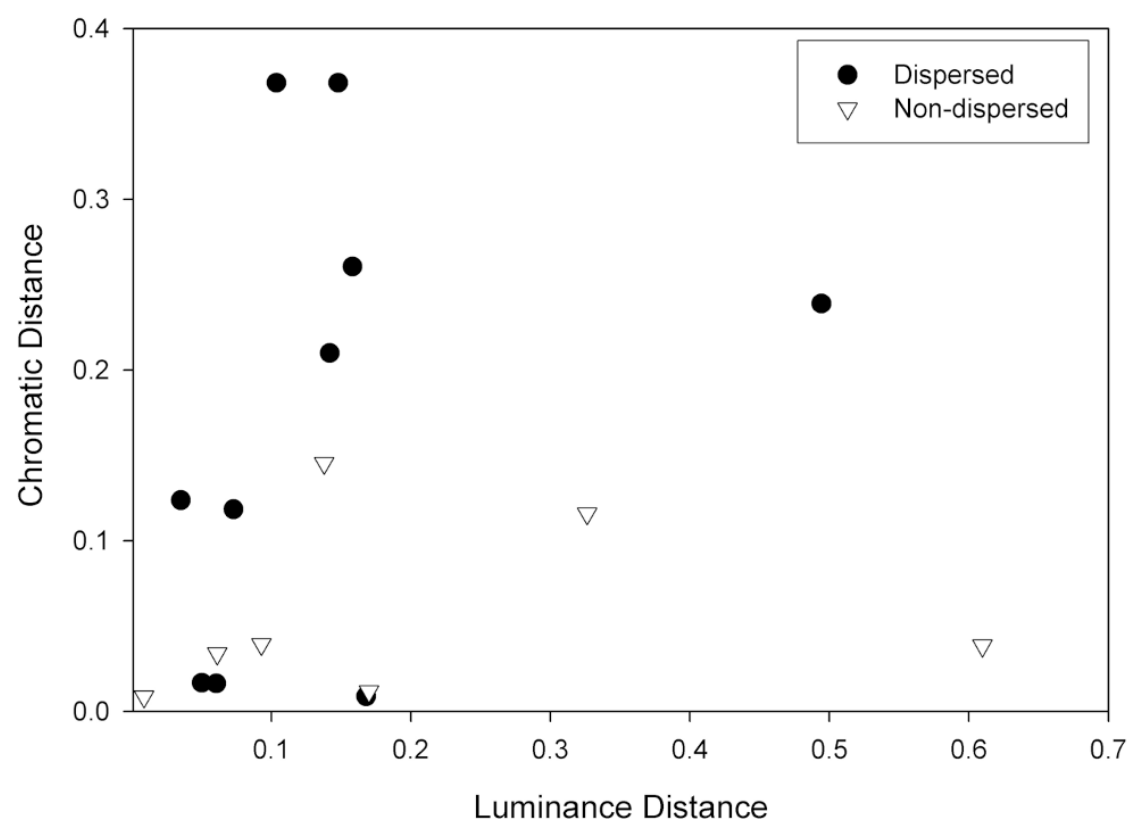

Figure $1 \mid$ Chromatic and luminance contrasts of dispersed and non-dispersed fruits. Scatterplot showing the blue-yellow chromatic contrasts (y axis) and luminance contrasts ( $\mathrm{x}$ axis) between ripe fruits and upper leaf surfaces of dispersed and non-dispersed fruits. Reflectance spectra of ripe fruits and upper leaf surfaces were measured relative to a Spectralon white reflectance standard using a Jaz portable spectrometer and a PX-2 pulsed xenon lamp emitting a D-65 light source. The chromatic and luminance conspicuity of food items was modeled as a ratio of the quantum catch of photons incident on the retina by different cone types, using a dichromatic visual model based on the long-wavelength sensitive (L) photopigments $\left(\lambda_{\text {max }} 558\right.$ nm) and short-wavelength sensitive (S) photopigments $\left(\lambda_{\max } 409 \mathrm{~nm}\right)$ possessed by Microcebus spp. 


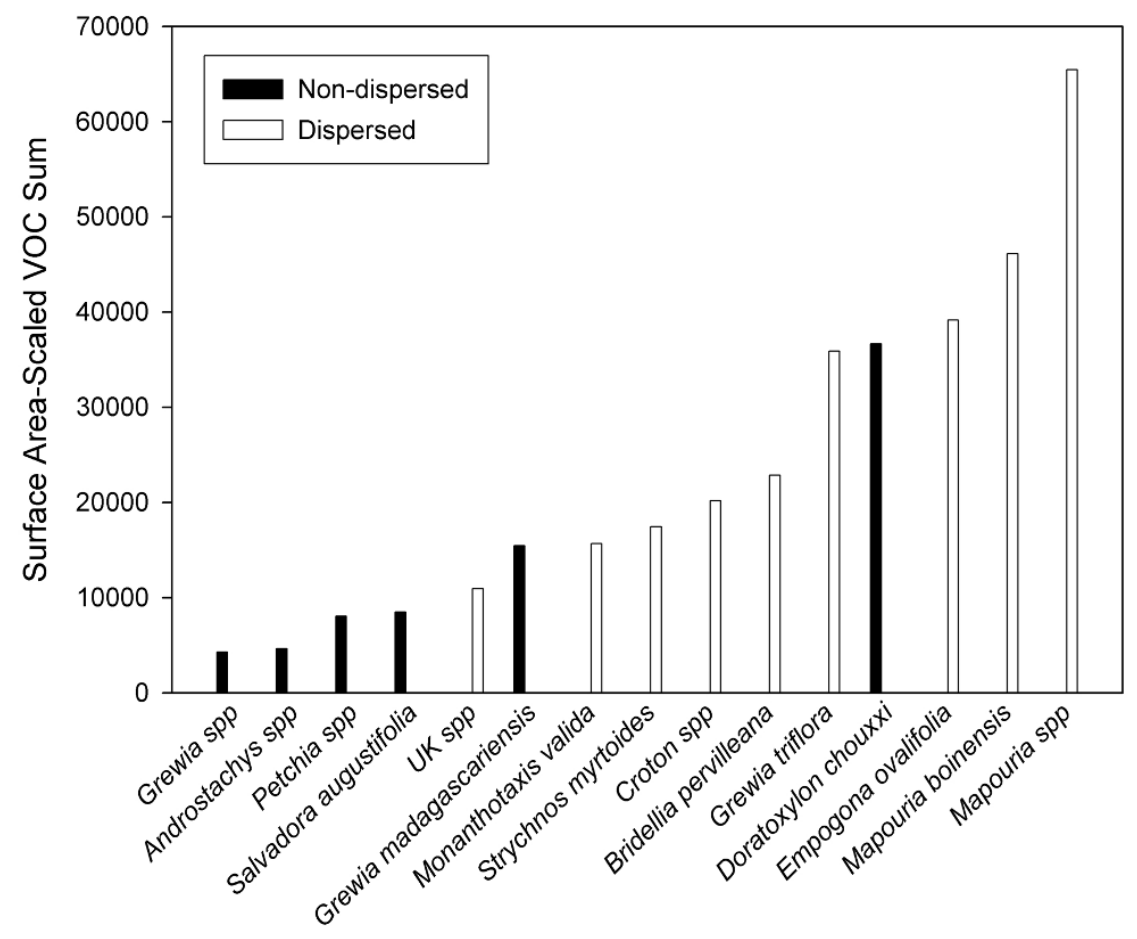

Figure $2 \mid$ Volatile organic compound emission intensity of dispersed and non-dispersed fruits. Frequency distribution of surface-area-scaled volatile organic compound (VOC) emissions of dispersed and non-dispersed fruits. Surface-area-scaled VOC emission intensity is determined by integrating areas under gas chromatography-mass spectrometry (GC-MS) chromatograms, and scaling GC-MS chromatograms by the total surface area of all fruits sampled.

suggest that the persistence of two functional opsin genes in some nocturnal primate species indicates that dichromatic colour vision is under purifying natural selection, and confers a foraging advantage $^{33,35}$. Our findings that under moonlight conditions - which are bright enough to support cone function ${ }^{36}$ - Microcebus-dispersed fruits display greater blue-yellow chromatic contrast from leaves than non-dispersed fruits supports the adaptive function of dichromatic colour vision for this genus.

Our prediction that individual VOCs predict fruit consumption and seed dispersal was not supported. Rather we found no relationship between the top ten most common VOCs and fruit consumption or seed dispersal. While our results are therefore not in accordance with the role of specific compounds during fruit selection, previous studies on other mammals support this relationship $^{13,18}$. There are at least two potential explanations for this discrepancy. First, because olfactory receptor gene repertoires have not yet been sequenced for this genus, Microcebus' phenotypic sensitivity to specific VOCs is unknown. Ideally, future work will be aimed at establishing olfactory sensitivity to specific compounds which would allow for a biologically meaningful approach to the question of fruit signal VOC specificity. Second, it is possible that Microcebus, like other olfactory-driven foragers ${ }^{2}$, are responsive to VOCs that are present even in trace amounts. Thus, rather than identifying the most common VOCs present in fruits, it would be beneficial to identify Microcebus' sensitivity to certain VOCs, in addition to identifying their presence at critical thresholds in fruits.

Our prediction that overall VOC emission intensity predicts fruit consumption and seed dispersal is supported, which is consistent with the expectation that Microcebus spp. rely heavily on olfaction ${ }^{32}$. Microcebus spp. have been shown experimentally to be able to reliably distinguish olfactory cues, and retain extensive neuroanatomical structures associated with enhanced olfactory discrimination ${ }^{19,32}$, which are useful for identifying fruit signals and cues in the wild. Our finding is consistent with results for other nocturnal mammals that have been found to distinguish between ripe and unripe fruit based solely on olfactory cues ${ }^{3}$. For example, one study of fruit bats found that they were able to reliably select ripe fruit based on the presence and intensity of VOCs ${ }^{13}$. Another study compared VOC emissions of bird- and bat-dispersed fig fruits, and found that figs dispersed primarily by olfactorily-driven bats emitted higher overall VOCs than figs dispersed primarily by visually-oriented birds ${ }^{8}$. That olfactory cues may be tightly linked to the foraging effectiveness of a nocturnal primate makes evolutionary sense - animals functioning in low ambient light environments can be expected to rely on nonvisual signals and cues during foraging.

Microcebus spp. are active under nocturnal conditions that are sub-optimal for colour vision, yet our study reveals that fruit chromaticity still informs some foraging decisions. Therefore the physical properties of their diet may directly contribute to the maintenance of dichromacy in Microcebus spp. while it has been lost in other nocturnal primates ${ }^{30,37}$. Though the activity patterns and resulting sensory adaptations of early primates is contested ${ }^{38}$, olfaction and colour vision have traditionally been portrayed as antagonistic, such that the advent of enhanced visual specializations is expected to co-vary with a decrease in reliance on olfaction ${ }^{17}$. Yet the variable patterns of loss and retention of vision and olfaction in different lineages under similar ecological pressures (i.e. nocturnality) reveals the details are more nuanced than this and need to be considered more carefully to identify relationships between diet, activity patterns and sensory systems.

Microcebus potentially represent their own seed disperser niche in the frugivore communities of Madagascar, as they are the only small bodied nocturnal frugivores that do not hibernate for most of the year. Because of their small body size they are restricted to dispersing seeds normally available to birds with opposite foraging patterns and sensory phenotypes. While avian seed dispersers are tetrachromatic and diurnal, and rely heavily on visual cues during foraging, Microcebus spp. are capable of fewer chromatic distinctions but have improved olfactory capabilities than sympatric, frugivorous birds. The difference in plant signals implied by these two sets of conflicting 
adaptations predicts functional separation in small fruit morphology. The co-occurrence of two small-seeded disperser guilds with differing sensory abilities is likely to result in different selective pressures on small fruit morphology, favoring both chromatic conspicuity that attracts highly visual diurnal birds, and VOC emissions and blue-yellow chromatic contrasts that attract olfactory-driven, dichromatic primates. Future research on the role of sensory phenotypes of Malagasy avian dispersers during foraging will help to illustrate the degree to which these adaptations either integrate or conflict.

\section{Methods}

Data were collected adjacent to Ampijoroa forestry station in the tropical dry forest in Ankarafantsika National Park, northwestern Madagascar (ANP - 15" $59^{\prime}-16^{\circ} 22 \mathrm{~S}$, $47^{\prime \prime} 56^{\prime}-47^{\prime \prime} 12 \mathrm{E}$ ). Over a three month period (May-July, 2012), we opportunistically collected ripe fruits of 20 species found growing within the study area. We offered a minimum of 40 individual ripe fruits $(\mathrm{N}=676)$ of 20 plant species to wild-trapped Microcebus $(\mathrm{N}=99)$ held in short-term captivity $(\sim 12 \mathrm{hrs})$ and identified and counted all seeds contained in feces collected from traps $(\mathrm{N}=1324)$. Only fruit species that contain seeds where the mean size is equal to or lesser than $11 \mathrm{~mm}$ in maximum diameter, which is the largest maximum diameter found in Microcebus fecal samples, were included in the analysis $(\mathrm{N}=16)$. A fruit species was considered to be dispersed when seeds of that species were present in feces. A fruit species was considered to be non-dispersed when a minimum of 40 fruits of that species were offered to captive Microcebus, and neither consumed nor discovered in Microcebus feces. In all cases but one (Monanthotaxis valida, Annonaceae), all fruit species were dispersed by both species of Microcebus. This research adhered to the Laws of Madagascar governing primate research, the American Society of Primatologists principles for the ethical treatment of primates, and the University of Toronto (Animal Care Protocol \#20009283).

To quantify fruit odour, fruits were collected in the field, and measured in the laboratory in three dimensions (height, width and depth) using sliding calipers, and placed inside plastic sampling bags (Reynold's large oven bags). The atmosphere within each bag was sampled using a vacuum pump (Gilian 5000, Sensidyne), which pulled air through the sample bag (1 L/min, $240 \mathrm{~min})$ and into two odourantabsorbent filters (Amberlite XAD-2, 400-200 mg, Sigma-Aldrich). Contamination of the sampling enclosure with ambient VOCs was minimized by passing incoming air through a container of activated carbon.

In order to analyze the trapped VOCs, XAD resin beds were removed from their cartridges and shaken manually in $4 \mathrm{~mL}$ hexane (Sigma Aldrich) for $5 \mathrm{~min}$. Main and breakthrough XAD beds were extracted separately. Extracts were analyzed using an Agilent 7890A gas chromatograph interfaced with an Agilent 5975 inert mass spectrometer operating in electron ionization (EI) mode. All injection volumes were $1 \mu \mathrm{L}$ and performed in the splitless mode with an inlet temperature of $250^{\circ} \mathrm{C}$. Separation was achieved using an Agilent DB- 5 column $(30 \mathrm{~m} \times 0.25 \mathrm{~mm} \times$ $0.25 \mu \mathrm{m})$ at a constant helium flow rate of $1 \mathrm{~mL} / \mathrm{min}$. The oven program consisted of an initial hold at $50^{\circ} \mathrm{C}$ for $2 \mathrm{~min}$ followed by a $10^{\circ} \mathrm{C} / \mathrm{min}$ ramp to $150^{\circ} \mathrm{C}$ and a $30^{\circ} \mathrm{C} /$ min ramp to $300^{\circ} \mathrm{C}$. The transfer line temperature was held at $300^{\circ} \mathrm{C}$. Analytes were monitored in full scan mode using a selected mass range of 40-300 Da.

In order to control for variation in fruit number and fruit surface area, we scaled the GC-MS chromatograms by the total surface area of all fruits in the sample bag. We determined total VOC emission intensity for each fruit species by summing the area under the surface-scaled GC-MS chromatograms, and compared values for dispersed and non-dispersed species with a one-way analysis of variance (ANOVA). Two fruit species (Elaeocarpus subserratus, Elaeocarpaceae, and Psorospermum crassifolia, Hypericaceae) were excluded from the analysis because samples were run using a different GC-MS, and thus were not quantitatively comparable. We determined the ten largest compound peaks for each fruit species and tentatively identified the five compounds that appear to be driving variation using MassLynx software (V4.1). To determine the effect of individual VOCs on fruit choice and seed dispersal, we analysed the largest ten VOC values in both dispersed and non-dispersed fruits using a one-tailed generalized linear model (GLM) with a log link function. We computed the Wald Likelihood statistic (SPSS V20), for both dispersed and non-dispersed categories, to test whether the shape of the distribution is significantly different from a Poisson distribution.

Reflectance spectra of ripe fruits (targets) and upper leaf surfaces (backgrounds) were measured relative to a Spectralon white reflectance standard (Labsphere) onsite in Madagascar using a Jaz portable spectrometer and a PX-2 pulsed xenon lamp (Ocean Optics Inc.) emitting a D-65 light source. The fruit scanning angle was fixed at $45^{\circ}$, and external light was blocked using thick black fabric. The chromatic and luminance conspicuity of food items was modeled as a ratio of the quantum catch of photons incident on the retina by different cone types following established methods ${ }^{12,39,40}$, using a dichromatic visual model based on the longwavelength sensitive (L) photopigments $\left(\lambda_{\max } 558 \mathrm{~nm}\right)$ and short-wavelength sensitive (S) photopigments $\left(\lambda_{\max } 409 \mathrm{~nm}\right)$ possessed by Microcebus ${ }^{30}$. The quantum catches of each photoreceptor (cone) type were calculated according to the formula:

$$
Q i=\int_{\min }^{\max } R(\lambda) I(\lambda) S i(\lambda) d \lambda
$$

where $\mathrm{Q}_{\mathrm{i}}$ represents the quantum catch of a photoreceptor $i$ over the range of the primate visual spectrum, from $400 \mathrm{~nm}(\min )$ to $700 \mathrm{~nm}(\max ), \mathrm{R}(\lambda)$ represents the reflectance spectrum, $I(\lambda)$ represents the irradiance spectrum, and $S_{i}(\lambda)$ is the spectral sensitivity function of the $i$-th photoreceptor (containing S or L photopigments). For the representative irradiance spectrum, we used down-welling moonlight in a large forest gap ${ }^{36}$. The spectral sensitivity function for each photoreceptor type was calculated as per Hiramatsu et al. ${ }^{12}$, with one alteration. Because lemurs do not possess a macula lutea, our pre-receptoral filter included only the effects of the lens, as opposed to the combined effects of the lens and macular pigment. Although the rods may contribute to colour perception at dim light levels, the perceptual effects of this are not well understood, and we omit the contribution of rods here for simplicity.

The blue-yellow chromaticities of target and background objects can be represented and plotted as the relative quantum catches of the $\mathrm{S}$ cones to the $\mathrm{L}$ cones, $\mathrm{S} / \mathrm{L}$. Because the $S$ cones do not contribute meaningfully to perception of luminance contrast, the relative luminance value of each object was estimated by dividing the quantum catch of the $L$ cones by that of a hypothetical white surface that reflects $100 \%$ of the illuminant. To estimate the blue-yellow chromatic contrast (BY) and the luminance contrast (LUM) between each target fruit and its respective leaf background, we calculated a contrast value for each channel: $\mathrm{BY}=\left|\ln \left(\mathrm{Q}_{L}^{\mathrm{f}}\right)-\ln \left(\mathrm{Q}_{L}^{\mathrm{b}}\right)\right|-$ $\left|\ln \left(\mathrm{Q}_{S}{ }^{\mathrm{f}}\right)-\ln \left(\mathrm{Q}_{S}^{\mathrm{b}}\right)\right| ; \mathrm{LUM}=\left|\ln \left(\mathrm{Q}_{L}{ }^{\mathrm{f}}\right)-\ln \left(\mathrm{Q}_{L}{ }^{\mathrm{b}}\right)\right|$, where $\mathrm{Q}$ denotes the quantum catch of the $\mathrm{L}$ cones $(\mathrm{L})$ or $\mathrm{S}$ cones $(\mathrm{S})$ for fruits (f) or backgrounds $(\mathrm{b})^{12}$.

To determine the effect of luminance and chromaticity on fruit choice and seed dispersal, we analyzed the differences between the leaves and ripe fruits using a onetailed GLM for binomial distribution with a logit link function and Wald Likelihood statistic (SPSS V20).

1. Schaefer, H., Schaefer, V. \& Vorobyev, M. Are fruit colors adapted to consumer vision and birds equally efficient in detecting colorful signals? The American Naturalist 169, S159-S169 (2007).

2. Linn Jr, C. E. et al. Postzygotic isolating factor in sympatric speciation in Rhagoletis flies: reduced response of hybrids to parental host-fruit odors. Proceedings of the National Academy of Sciences 101, 17753-17758 (2004).

3. Korine, C. \& Kalko, E. K. V. Fruit detection and discrimination by small fruiteating bats (Phyllostomidae): echolocation call design and olfaction. Behavioral Ecology and Sociobiology 59, 12-23 (2005).

4. Hirsch, B. T. Tradeoff between travel speed and olfactory food detection in ringtailed coatis (Nasua nasua). Ethology 116, 671-679 (2010).

5. Valido, A., Schaefer, H. M. \& Jordano, P. Colour, design and reward: phenotypic integration of fleshy fruit displays. Journal of Evolutionary Biology 24, 751-760 (2011).

6. Schaefer, H. \& Braun, J. Reliable cues and signals of fruit quality are contingent on the habitat in black elder (Sambucus nigra). Ecology 90, 1564-1573 (2009).

7. Otte, D. Effects and functions in the evolution of signaling systems. Annual Review of Ecology and Systematics 5, 385-417 (1974).

8. Lomáscolo, S., Levey, D., Kimball, R., Bolker, B. \& Alborn, H. Dispersers shape fruit diversity in Ficus (Moraceae). Proceedings of the National Academy of Sciences 107, 14668-14672 (2010).

9. Dominy, N. J. \& Lucas, P. W. Ecological importance of trichromatic vision to primates. Nature 410, 363-366 (2001).

10. Lomáscolo, S. \& Schaefer, H. Signal convergence in fruits: a result of selection by frugivores? Journal of Evolutionary Biology 23, 614-624 (2010).

11. Melin, A. D., Fedigan, L. M., Hiramatsu, C. \& Kawamura, S. Polymorphic color vision in white-faced capuchins (Cebus capucinus): Is there foraging niche divergence among phenotypes? Behavioral Ecology and Sociobiology 62, 659-670 (2008).

12. Hiramatsu, C. et al. Importance of achromatic contrast in short-range fruit foraging of primates. PLoS ONE 3, e3356 (2008).

13. Sanchez, F. et al. Ethanol and methanol as possible odor cues for egyptian fruit bats (Rousettus aegypticus). Journal of Chemical Ecology 32, 1289-1300 (2006).

14. Osorio, D. \& Vorobyev, M. A review of the evolution of animal colour vision and visual communication signals. Vision Research 48, 2042-2051 (2008).

15. Rouquier, S. \& Giorgi, D. Olfactory receptor gene repertoires in mammals. Mutat Res 616, 95-102 (2007).

16. Dambroski, H. R. et al. The genetic basis for fruit odor discrimination in Rhagoletis flies and its significance for sympatric host speciation. Evolution 59, 1953-1964 (2005).

17. Gilad, Y., Wiebe, V., Przeworski, M., Lancet, D. \& Paabo, S. Loss of olfactory receptor genes coincides with the acquisition of full trichromatic vision in primates. PLoS Biology 2, 120-125 (2004).

18. Laska, M., Liesen, A. \& Teubner, P. Enantioselectivity of odor perception in squirrel monkeys and humans. American Journal of Physiology 277, 1098-1103 (1999).

19. Barton, R. A., Purvis, A. \& Harvey, P. H. Evolutionary radiation of visual and olfactory brain systems in primates, bats and insectivores. Philosophical Transactions of the Royal Society of London B 348, 381-392 (1995). 
20. Hiramatsu, C. et al. Interplay of olfaction and vision in fruit foraging of spider monkeys. Animal Behaviour 77, 1421-1426 (2009).

21. Melin, A. D. et al. Fig foraging by dichromatic and trichromatic Cebus capucinus in a tropical dry forest. International Journal of Primatology 30, 753-775 (2009).

22. Rushmore, J., Leonhardt, S. D. \& Drea, C. M. Sight or scent: Lemur sensory reliance in detecting food quality varies with feeding ecology. PLoS One 7, 1-11 (2012).

23. Garber, P. A. \& Lambert, J. E. Primates as seed dispersers: Ecological processes and directions for future research. American Journal of Primatology 45, 3-8 (1998).

24. Lambert, J. E. \& Garber, P. A. Evolutionary and ecological implications of primate seed dispersal. American Journal of Primatology 45, 9-28 (1998).

25. Chapman, C. A. \& Chapman, L. J. Survival without dispersers: seedling recruitment under parents. Conservation Biology 9, 675-678 (1995)

26. Lambert, J. E. Seed handling in chimpanzees (Pan troglodytes) and redtail monkeys (Cercopithecus ascanius): implications for understanding hominoid and cercopithecine fruit-processing strategies and seed dispersal. American Journal of Physical Anthropology 109, 365-386 (1999).

27. Lambert, J. E. Primate frugivory and seed dispersal: Implications for the conservation of biodiversity. Evolutionary Anthropology 19, 165-166 (2010).

28. Wright, P. C. et al. Frugivory in four sympatric lemurs: Implications for the future of Madagascar's forests. American Journal of Primatology 73, 585-602 (2011).

29. Ganzhorn, J. U. et al. Possible fruit protein effects on primate communities in Madagascar and the neotropics. Plos One 4, 1-8 (2009).

30. Tan, Y. \& Li, W. H. Trichromatic vision in prosimians. Nature 402, 36 (1999)

31. Dkhissi-Bentahya, O., Szel, A., Degrip, W. J. \& Cooper, H. M. Short and midwavelength cone distribution in a nocturnal strepsirrhine primate (Microcebus murinus). The Journal of Comparative Neurology 438, 490-504 (2001).

32. Siemers, B. M. et al. Sensory basis of food detection in wild Microcebus murinus. International Journal of Primatology 28, 291-304 (2007).

33. Veilleux, C. C., Louis, E. E. \& Bolnick, D. A. Nocturnal light environments influence color vision and signatures of selection on the OPN1SW opsin gene in nocturnal lemurs. Molecular Biology and Evolution (In Press).

34. Morgan, M. J., Adam, A. \& Mollon, J. D. Dichromats detect colour-camouflaged objects that are not detected by trichromats. Proceedings: Biological Sciences 248, 291-295 (1992).

35. Perry, G. H., Martin, R. D. \& Verrelli, B. C. Signatures of functional constraint at aye-aye opsin genes: The potential of adaptive color vision in a nocturnal primate. Molecular Biology and Evolution 24, 1963-1970 (2007).

36. Melin, A. D., Moritz, G. L., Fosbury, R. A., Kawamura, S. \& Dominy, N. J. Why aye-ayes see blue. American journal of Primatology 74, 185-192 (2012).

37. Jacobs, G. H. Losses of functional opsin genes, short-wavelength cone photopigments, and color vision - A significant trend in the evolution of mammalian vision. Visual Neuroscience 30, 39-53 (2013).
38. Sussman, R. W., Rasmussen, T. \& Raven, P. H. Rethinking primate origins again. American Journal of Primatology 75, 95-106 (2013).

39. Sumner, P. \& Mollon, J. D. Chromaticity as a signal of ripeness in fruits taken by primates. The Journal of Experimental Biology 203, 1987-2000 (2000).

40. Osorio, D., Smith, A. C., Vorobyev, M. \& Buchanan-Smith, H. M. Detection of fruit and the selection of primate visual pigments for color vision. American Naturalist 164, 696-708 (2004).

\section{Acknowledgements}

We thank MICET and Madagascar National Parks, for permission to conduct this research in Madagascar. We thank Dr. Scott Mabury for the loan of instrumentation. We are grateful to Mr. Paul Tsiveraza, Tantely, Jhonny, Radoniaina Rafaliarison and Jean de-la-Dieu for contributions in the field. We thank Sharon Kessler, Cindy Canale, Ute Radespiel, Blanchard Randrianambinina and Sylvia Lomascolo for their help in the initial phases of this project. We appreciate assistance from Dr. Chihiro Hiramatsu and Dr. James Higham with aspects of the colour modelling. For helpful commentary, we thank C.J. Toborowsky and Paul R. Duffy. We are grateful to Dr. Michael Huffman, Dr. James Higham, and an anonymous reviewer for valuable comments on this manuscript. This research adhered to the Laws of Madagascar governing primate research, the American Society of Primatologists principles for the ethical treatment of primates, and the University of Toronto (Animal Care Protocol \#20009283). For funding we thank Sigma Xi, GM Women in Science (K.V.), the University of Toronto (K.V. and R.J.B.) and Natural Sciences and Engineering Research Council of Canada (K.V., R.J.B., A.D.M., S.M.L.)

\section{Author contributions}

K.V., R.J.B. and A.D.M. designed the study. K.V. and R.J.B. carried out data collection. K.V., S.A.S. and D.A.J. designed the VOC collection system, and extracted and analyzed chemical data. A.D.M. completed colour modeling and analysis. R.J.B. and S.M.L. carried out all statistical analyses. All authors contributed to the writing and editing of the manuscript.

\section{Additional information}

Competing financial interests: The authors declare no competing financial interests.

How to cite this article: Valenta, K. et al. Colour and odour drive fruit selection and seed dispersal by mouse lemurs. Sci. Rep. 3, 2424; DOI:10.1038/srep02424 (2013).

This work is licensed under a Creative Commons AttributionNonCommercial-NoDerivs 3.0 Unported license. To view a copy of this license, visit http://creativecommons.org/licenses/by-nc-nd/3.0 